\title{
Occurrence of LINER galaxies within the galaxy group environment
}

\author{
Georgina V. Coldwell, ${ }^{1 \star}$ Luis Pereyra, ${ }^{2}$ Sol Alonso, ${ }^{1 \star}$ Emilio Donoso ${ }^{3}$ \\ and Fernanda Duplancic ${ }^{1}$ \\ ${ }^{1}$ Departamento de Geofísica y Astronomía, CONICET, Facultad de Ciencias Exactas, Físicas y Naturales, Universidad Nacional de San Juan, Av. Ignacio de \\ la Roza 590 (O), J5402DCS Rivadavia, San Juan, Argentina \\ ${ }^{2}$ Instituto de Astronomía Teórica y Experimental, CONICET, Observatorio Astronómico, Universidad Nacional de Córdoba, X5000BGR Córdoba, Argentina \\ ${ }^{3}$ Instituto de Ciencias Astronómicas de la Tierra y el Espacio, CONICET, Universidad nacional de San Juan, CC49, 5400 San Juan, Argentina
}

Accepted 2017 January 31. Received 2017 January 6; in original form 2016 October 14

\begin{abstract}
We study the properties of a sample of 3967 low-ionization nuclear emission-line region (LINER) galaxies selected from SDSS-DR7, with respect to their proximity to galaxy groups. The host galaxies of LINERs have been analysed and compared with a well-defined control sample of 3841 non-LINER galaxies matched in redshift, luminosity, colour, morphology, age and stellar mass content. We find no difference between LINER and control galaxies in terms of the colour and age of stellar population as a function of the virial mass and distance to the geometric centre of the group. However, we find that LINERs are more likely to populate lowdensity environments in spite of their morphology, which is typical of high-density regions such as rich galaxy clusters. For rich (poor) galaxy groups, the occurrence of LINERs is approximately two times lower (higher) than the occurrence of matched, non-LINER galaxies. Moreover, LINER hosts do not seem to follow the expected morphology-density relation in groups of high virial mass. The high frequency of LINERs in low-density regions could be due to the combination of a sufficient gas reservoir to power the low-ionization emission and/or enhanced galaxy interaction rates benefiting the gas flow towards their central regions.
\end{abstract}

Key words: galaxies: active-galaxies: general-galaxies: groups: general-galaxies: statistics.

\section{INTRODUCTION}

Many features present in galaxy spectra, usually characterized by the intensity of absorption or emission lines, shape of continuum, etc., can provide important information about their formation and evolution. In particular, emission lines probe the gaseous and chemical components of galaxies. The low-ionization nuclear emissionline regions (LINERs) were described by Heckman (1980) as a class of extragalactic objects with optical spectra dominated by enhanced low-ionization $\mathrm{O} I(\lambda 6300)$ and $\mathrm{N}$ II $(\lambda 6548,6583)$ lines. Thus, LINERs were defined by intensity ratios of optical emission lines, namely (1) $I([\mathrm{O}$ II $] \lambda 3727) / I\left([\mathrm{O}\right.$ III] $\lambda 5007) \geq 1$, where $\left[\mathrm{O}_{\mathrm{II}}\right]$ $\lambda 3727$ is used to designate the [O II] $\lambda \lambda 3726,3729$ doublet; and (2) $I([\mathrm{O}$ I $] \lambda 6300) / I([\mathrm{O}$ III $] \lambda 5007) \geq 1 / 3$.

The nature of the ionization source that powers emission lines has not been determined. In the last three decades, different scenarios for the LINER excitation mechanism have been proposed. The most feasible are (1) ionization by shock-heated gas due to direct mechanical energy input of turbulent gas motions, jets or bubbles (Heckman 1980; Dopita \& Sutherland 1995); (2) stellar pho-

^E-mail: georginacoldwell@gmail.com (GVC); solalonsog@gmail.com (SA) toionization by hot O stars (Filipenko \& Terlevich 1992) or by old post-asymptotic giant branch (post-AGB) stars (Binette et al. 1994; Stasínska et al. 2008); and (3) photoionization by an active central black hole (Groves, Dopita \& Sutherland 2004), such as an active galactic nucleus (AGN).

The first mechanism, i.e. gas heated by fast shock waves, is probably the less favoured scenario because the velocity dispersion of nuclear gas commonly falls below the value required to explain the observed level of spectral ionization (Ho, Filippenko $\&$ Sargent 2003). However, there is further controversy related to the last two mechanisms proposed. Observations at radio (Nagar, Falcke \& Wilson 2005) and X-ray wavelengths (González-Martín et al. 2009a) provide strong support for an AGN as the origin of LINER emission. LINERs seem to populate the low-luminosity end of the AGN distribution (Kewley et al. 2006), where radiatively inefficient accretion flows and external obscuring matter (Dudik, Satyapal \& Marcu 2009; González-Martín et al. 2009b) may cause the optical extinction. Thus, LINERs are less luminous than Seyfert galaxies and share similar spectral characteristics, with the notable exception that LINERs show enhanced low-ionization $\mathrm{O}$ I $(\lambda 6300)$ and $\mathrm{N}$ II $(\lambda 6548,6583)$ lines (Heckman 1980). Furthermore, there is new evidence supporting the hypothesis of stellar photoionization, as some authors suggest that hot post-AGB stars and white dwarfs could provide enough ionization to explain the LINER emission 
(Binette et al. 1994; Sodré \& Stasińska 1999; Stasínska et al. 2008). These stellar ionization sources are located in spatially extended regions around the nucleus as the $\mathrm{H} \alpha$ and $\mathrm{H} \beta$ brightness profiles do not decrease with $r^{-2}$, i.e. the radial dependence expected for a central ionization source such as an AGN (Sarzi et al. 2010; Yan \& Blanton 2012).

LINERs seem to be common phenomena, and they have been found in about 30 per cent of nearby galaxies (Heckman 1980; Ho, Filippenko \& Sargent 1997). This percentage increases for galaxies with early-type morphology, reaching 50 per cent in elliptical passive galaxies (Phillips et al. 1986; Goudfrooij et al. 1994; Yan et al. 2006; Capetti \& Baldi 2011). This early galaxy type is consistent with that residing in dense galaxy environments. The well-known morphology-age-density relation (Dressler 1980; Domínguez, Muriel \& Lambas 2001) shows that red and massive early-type galaxies are associated with rich galaxy structures preferably located in the cores of groups or clusters of galaxies. On the other hand, late-type and blue galaxies are typically found in the outer regions of galaxy groups or in the field.

Since galaxy evolution correlates with different environmental factors, the study of the neighbourhood of LINERs could provide important clues about the nature of their low-ionization emission. If LINERs are dominated by star-forming $\mathrm{H}$ II regions, we would expect them to follow the morphology-density segregation (Dressler 1980). Instead, several recent studies have found that the AGN environment does not seem to follow this relation (Popesso \& Biviano 2006; Coldwell et al. 2009; Padilla, Lambas \& González 2010; Coldwell et al. 2014). Then, if the presence of an AGN is the main cause of the LINER emission, we could assume that LINERs will follow a similar trend to, for example, Seyfert 2 galaxies.

In addition, it is not clear that LINERs constitute a homogeneous class of objects. Unlike galaxies dominated by star-forming H II regions or Seyferts, whose ionization sources are clearly identified as young massive stars and AGNs, respectively, LINERs can be produced by a wide array of ionization mechanisms. For example, Hubble Space Telescope observations have found that both unresolved nuclear and extended $\mathrm{H} \alpha$ emissions are present in the majority of nearby LINERs (Masegosa et al. 2011), and LINERlike ratios have long been observed in extended emission-line regions (Heckman 1989; Sarzi et al. 2010). In addition, Graves et al. (2007) found that red-sequence galaxies with LINER emission are younger than their quiescent counterparts, suggesting a connection between the star formation history and the mechanism generating the low-ionization emission. But, there is no certainty about which mechanism prevails in LINER galaxies. So the true power source of LINER emission is still under debate, and most observations do not have resolution high enough to detect if this emission comes from extended or nuclear regions of the galaxies.

The aim of this paper is to study the relation between LINER objects and their local density environment in order to shed light on the origin of their main emission mechanism. The layout of this paper is as follows: In Section 2, we briefly describe the data selection and LINER classification scheme; in Section 3, we describe the properties of galaxies hosting LINERs, the procedure used to construct the control sample and their correlation with galaxy group parameters. In Section 4, we investigate the occurrence of LINERs in galaxy groups and their dependence on group proximity. Finally, in Section 5, we discuss the results and draw our conclusions. Throughout this paper, we have assumed a $\Lambda$-dominated cosmology, with $\Omega_{\mathrm{m}}=0.3, \Omega_{\lambda}=0.7$ and $H_{0}=$ $100 \mathrm{~km} \mathrm{~s}^{-1} \mathrm{Mpc}^{-1}$.

\section{DATA AND SAMPLE SELECTION}

In this paper, we used galaxy samples selected from the spectroscopic Sloan Digital Sky Survey Data Release 7 (SDSS-DR7; Abazajian et al. 2009). The SDSS-DR7 main galaxy sample is essentially a magnitude-limited catalogue (Petrosian 1976) with $r_{\text {lim }}<17.77$, consisting of $\sim 700000$ galaxies with measured spectra and photometry in five optical bands $(u, g, r, i, z)$, and with a median redshift of 0.1 (Strauss et al. 2002).

The procedures to estimate the physical properties of galaxies used in this analysis are described by Brinchmann et al. (2004), Tremonti et al. (2004) and Blanton et al. (2005). These data are available from MPA/JHU ${ }^{1}$ and $\mathrm{NYU},{ }^{2}$ including gas-phase metallicities, stellar masses, indicators of recent major starbursts, current total and specific star formation rates, emission-line fluxes and Sérsic indices among others.

\subsection{LINER selection}

For the AGN selection, we use the publicly available emission-line fluxes, whose measurement is detailed in Tremonti et al. (2004). Additionally, we have corrected for optical reddening using the Balmer decrement and the Calzetti et al. (2000) dust curve. We assume an $R_{V}=A_{V} / E(B-V)=3.1$ and an intrinsic Balmer decrement $(\mathrm{H} \alpha / \mathrm{H} \beta)_{0}=3.1$ (Osterbrock \& Miller 1989). Since the true uncertainties of the emission-line measurements were underestimated, the signal-to-noise ratio $(\mathrm{S} / \mathrm{N})$ of every line was calculated with the flux errors adjusted, as suggested by the MPA/JHU team. ${ }^{3}$

The emission-line galaxy sample was restricted to have a redshift range of $0.04<z<0.1$. The lower limit prevents small fixed-size apertures from affecting galaxy properties derived from the fibre spectra, and the upper limit corresponds to the luminosity completeness limit for the SDSS sample. Furthermore, we include only galaxies with $\mathrm{S} / \mathrm{N}>2$ for all the lines involved in the three diagnostic diagrams used to discriminate Seyfert 2 from star-forming and LINER galaxies. This conservative criterion only moderately reduces the effective sample size, yet assures a more reliable selection of LINER objects. Thus, from this refined sample, we separate Seyfert 2, LINER and star-forming galaxies using the three standard (Baldwin, Phillips \& Terlevich 1981, hereafter BPT) line-ratio diagrams. The AGN-starburst separation, as suggested by Kewley et al. (2001, 2006), depends on the relative source location within BPT diagnostic diagrams, and follows the equations

$$
\begin{aligned}
& \log ([\mathrm{O} I I I] / H \beta)>0.61 /\left(\log \left(\left[\mathrm{N}_{\mathrm{II}} / \mathrm{H} \alpha\right]\right)-0.47\right)+1.19, \\
& \log \left(\left[\mathrm{O}_{\mathrm{III}}\right] / \mathrm{H} \beta\right)>0.72 /\left(\log \left(\left[\mathrm{S}_{\mathrm{II}} / \mathrm{H} \alpha\right]\right)-0.32\right)+1.30, \\
& \log \left(\left[\mathrm{O}_{\mathrm{III}}\right] / \mathrm{H} \beta\right)>0.73 /\left(\log \left(\left[\mathrm{O}_{\mathrm{I}} / \mathrm{H} \alpha\right]\right)+0.59\right)+1.33
\end{aligned}
$$

LINERs are located below the Seyfert/LINER division line, as indicated by the equations

$$
\begin{aligned}
& \log ([\mathrm{O} I I I] / H \beta)<1.89 \log \left(\left[\mathrm{S}_{\mathrm{II}} / \mathrm{H} \alpha\right]\right)+0.76, \\
& \log \left(\left[\mathrm{O}_{\mathrm{III}}\right] / \mathrm{H} \beta\right)<1.18 \log \left(\left[\mathrm{O}_{\mathrm{I}} / \mathrm{H} \alpha\right]+1.30 .\right.
\end{aligned}
$$

On the grounds of internal consistency, we exclude from analysis ambiguous galaxies classified as one type of object in one BPT and

\footnotetext{
${ }^{1}$ http://www.mpa-garching.mpg.de/SDSS/DR7/

${ }^{2} \mathrm{http}: / /$ sdss.physics.nyu.edu/vagc/

${ }^{3}$ http://www.mpa-garching.mpg.de/SDSS/DR7/raw_data.html
} 


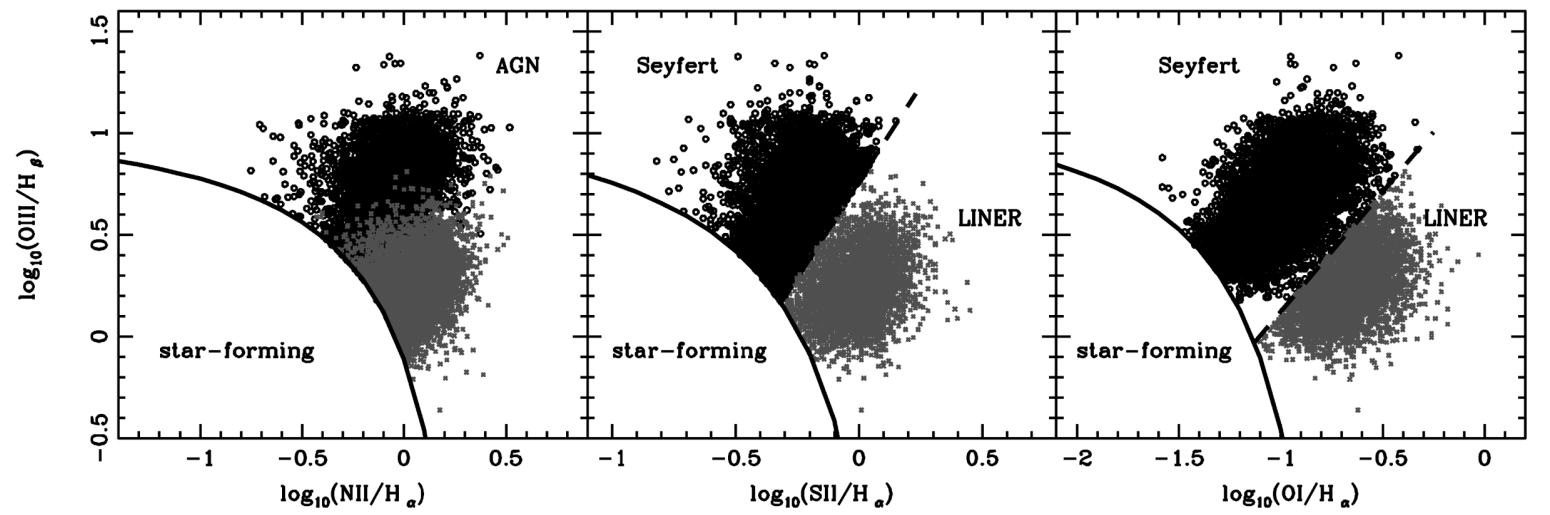

Figure 1. BPT diagrams of the selection criteria defined by Kewley et al. (2006) used to classify emission-line galaxies as Seyferts or LINERs. The panels show the line ratios $\log \left(\left[\mathrm{O}_{\text {III }}\right] / \mathrm{H} \beta\right)$ versus $\log \left([\mathrm{N}\right.$ II $/ \mathrm{H} \alpha]$ ) (left-hand panel) $\log \left(\left[\mathrm{O}_{\text {III }}\right] / \mathrm{H} \beta\right)$ versus $\log \left(\left[\mathrm{S}_{\text {II }} / \mathrm{H} \alpha\right]\right.$ (middle panel) and $\log ([\mathrm{O}$ III $] / \mathrm{H} \beta)$ versus $\log \left(\left[\mathrm{O}_{\mathrm{I}} / \mathrm{H} \alpha\right]\right.$ (right-hand panel). Seyferts are indicated by black dots and LINER galaxies by grey crosses. The solid lines separate star-forming galaxies from AGNs, and the dashed lines represent the Seyfert-LINER demarcation.

another in the remaining two diagrams. Bearing this in mind, we obtain an effective sample of 4778 LINER objects. The discriminated samples and the selection criteria are shown in the three BPT diagrams of Fig. 1.

\subsection{Galaxy group catalogue}

In this work, we use the group catalogues constructed by Zapata et al. (2009), which were identified by an improved version of the Huchra \& Geller (1982) friends-of-friends algorithm, with variable linking lengths of $D_{12}=D_{0} R$ and $V_{12}=V_{0} R$. These linking lengths are taken in the direction perpendicular and parallel to the line of sight, respectively. The spatial scaling $R$ takes into account the variation in the space density of galaxies in a flux-limited catalogue, and is calculated from the ratio of the density of galaxies brighter than the minimum required to enter the catalogue at the mean distance of the galaxies being linked to a characteristic survey depth. As found by Merchán \& Zandivarez (2005), the algorithm parameters $D_{0}=0.239 h^{-1} \mathrm{Mpc}$ and $V_{0}=450 \mathrm{~km} \mathrm{~s}^{-1}$ are selected to produce a reasonably complete sample (95 per cent) with low contamination $(<8$ per cent).

In addition, the virial mass of galaxy groups is computed by means of the virial theorem, given by

$M_{\mathrm{vir}}=\frac{3 \sigma_{v}^{2} r_{\mathrm{vir}}}{G}$

where $\sigma_{v}$ is the line-of-sight velocity dispersion and $r_{\text {vir }}$ is estimated as in Merchán \& Zandivarez (2005), i.e.

$r_{\mathrm{vir}}=\frac{\pi}{2} \frac{N_{\mathrm{g}}\left(N_{\mathrm{g}}-1\right)}{\sum_{i>j} r_{i j}^{-1}}$,

where $N_{\mathrm{g}}$ is the number of galaxy members and $r_{i j}$ are the relative projected distances between galaxies.

\section{HOST GALAXY PROPERTIES}

The morphology of a galaxy can be indicative of evolutionary processes it has been subjected to, and therefore indicative of its environment. Spiral galaxies are known to be relatively unevolved galaxies residing typically in low-density environments. On the other hand, elliptical galaxies are known to be more evolved sources, as a result of dynamical processes such as mergers, and known to inhabit high-density environments like galaxy groups or clusters
(Toomre \& Toomre 1972; Dressler 1997), where they are subjected to different effects such as strangulation, ram pressure and close encounters.

The LINER host galaxies have particular characteristics, which could give clues about their history. Fig. 2 shows the normalized distributions of (i) redshift; (ii) (Petrosian) $r$-band absolute magnitude (Blanton et al. 2003b), extinction- and $k$-corrected band shifted to $z=0.1$, using the software K-CORRECT_v4.2 of Blanton \& Roweis (2007); (iii) stellar mass in logarithmic scale, $M^{\star}$, previously determined by Kauffmann et al. (2003) ${ }^{4}$; (iv) $D_{\mathrm{n}}(4000)$ break index (Kauffmann \& Haehnelt 2002); (v) Sérsic index (Sérsic 1963); and (vi) colour $\left(M_{g}-M_{r}\right)$.

The break index $D_{\mathrm{n}}(4000)$ is defined as the ratio of the average flux density in the narrow continuum bands (3850-3950 and 4000$4100 \AA$ ) and is suitably correlated to the mean age of the stellar population in a galaxy and can be used to estimate the star formation rate (Brinchmann et al. 2004). The majority of star formation takes place preferentially in galaxies with low $D_{\mathrm{n}}(4000)$ values.

The change of surface brightness with distance from the galaxy centre can be well described by the Sérsic law, which has the form $\ln I(R)=\ln I_{0}-k R^{1 / n}$. Here, $I_{0}$ is the intensity at the centre and the Sérsic index $n$ controls the degree of curvature of the profile. Setting $n=4$ recovers a de Vaucouleurs profile, i.e. a good description of giant elliptical galaxies, while setting $n=1$ recovers the Freeman exponential profile, which is a good description of the light distribution in disc galaxies and dwarf ellipticals. Most galaxies are well fitted by Sérsic indices in the range of $0.5<n<10$.

Fig. 2 also shows the distributions corresponding to the SDSSDR7 main galaxy sample (within the same redshift range). The bimodality in the distribution of $D_{\mathrm{n}}(4000)$ and colour for the main galaxy sample is significantly noticeable. This effect is explained by the presence of two populations of galaxies in different stages of evolution: a population of young and blue galaxies, and another composed of old and red galaxies. It is expected that the mean and variance of both colour distributions are dependent on the luminosity function and stellar mass (Bernardi et al. 2003; Blanton et al. 2003a; Hogg et al. 2004) of the population considered. Moreover, Fig. 2 shows that most LINERs are hosted by a single population of red and old galaxies. Consistently, these LINER hosts are

\footnotetext{
${ }^{4}$ The method relies on spectral indicators relating to the stellar age and the fraction of stars formed in recent bursts.
} 

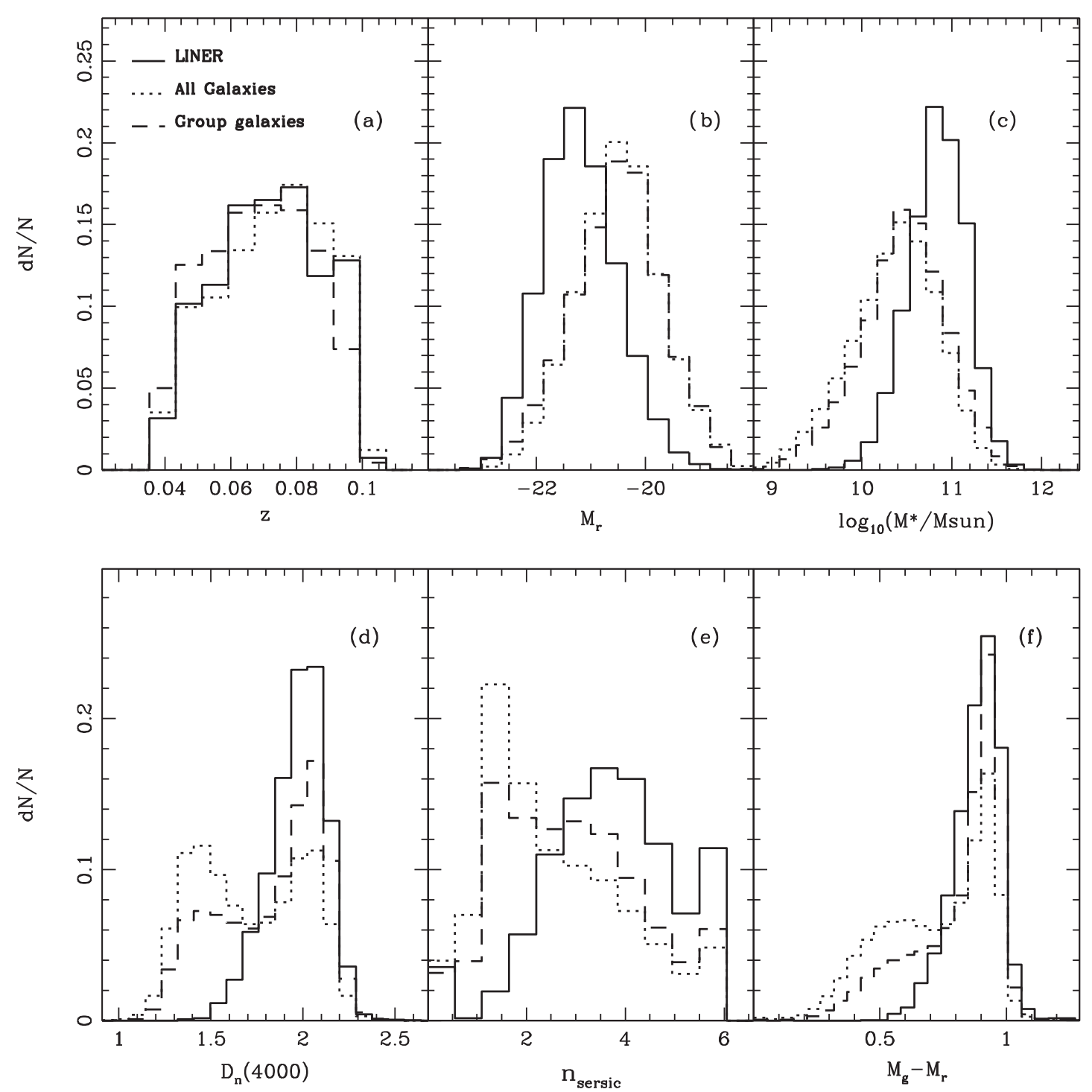

Figure 2. Normalized distributions of galaxy properties for SDSS-DR7 galaxies (dotted line), LINER total sample (solid line) and galaxy group members (dashed line).

significantly more massive and luminous than the general galaxy sample and have a bulge-type morphology.

These characteristics of LINERs are in agreement with the results of Kewley et al. (2006) and Capetti \& Baldi (2011), and are consistent with very well known features of galaxy groups and cluster galaxy members (Dressler 1980, 1984). We include in Fig. 2 the distributions of SDSS-DR7 galaxy groups, as constructed by Zapata et al. (2009), noting that galaxy group members show similar trends to the SDSS main sample, but with a higher percentage of red and old galaxies. This means that galaxy groups have a population of blue and young spiral galaxies, probably located in their outer regions, and another population of bright, red and bulge-type galaxies found in the centre of the systems. This is a clear consequence of the morphological segregation (Domínguez et al. 2001) of galaxies in clusters.

In this scenario, LINER galaxies can be thought of as galaxies lying in the central area of rich galaxy groups and clusters. However, after cross-correlating the LINER catalogue with the catalogue of galaxy groups, we found that only 12.4 per cent (592 objects) are members of galaxy groups with more than 10 members, within the redshift interval considered. Instead of that, 20.4 per cent of LINERs (974 objects) have been found to be members of galaxy groups consisting of four to nine members. This low percentage of LINERs belonging to galaxy groups consisting of more than 10 members motivates us to explore the relation between LINER properties and the group density environment.

\subsection{LINERs close to galaxy groups and construction of a control sample}

Galaxy groups are the most common structures in the universe, and the connection between LINERs and high-density environments could provide valuable information about the mechanisms responsible of their low-ionization emission.

Considering the low occurrence rate of LINERs in groups, we adopted LINERs belonging to galaxy groups as those objects with projected distances $r_{\mathrm{p}}<2 r_{\text {vir }}$, where $r_{\mathrm{vir}}$ is the virial radius, and the radial velocity difference $\Delta V<1000 \mathrm{~km} \mathrm{~s}^{-1}$, with respect to the geometric centre of the group. Thus, with this more relaxed criterion than only to be a galaxy group member, we are considering galaxies 

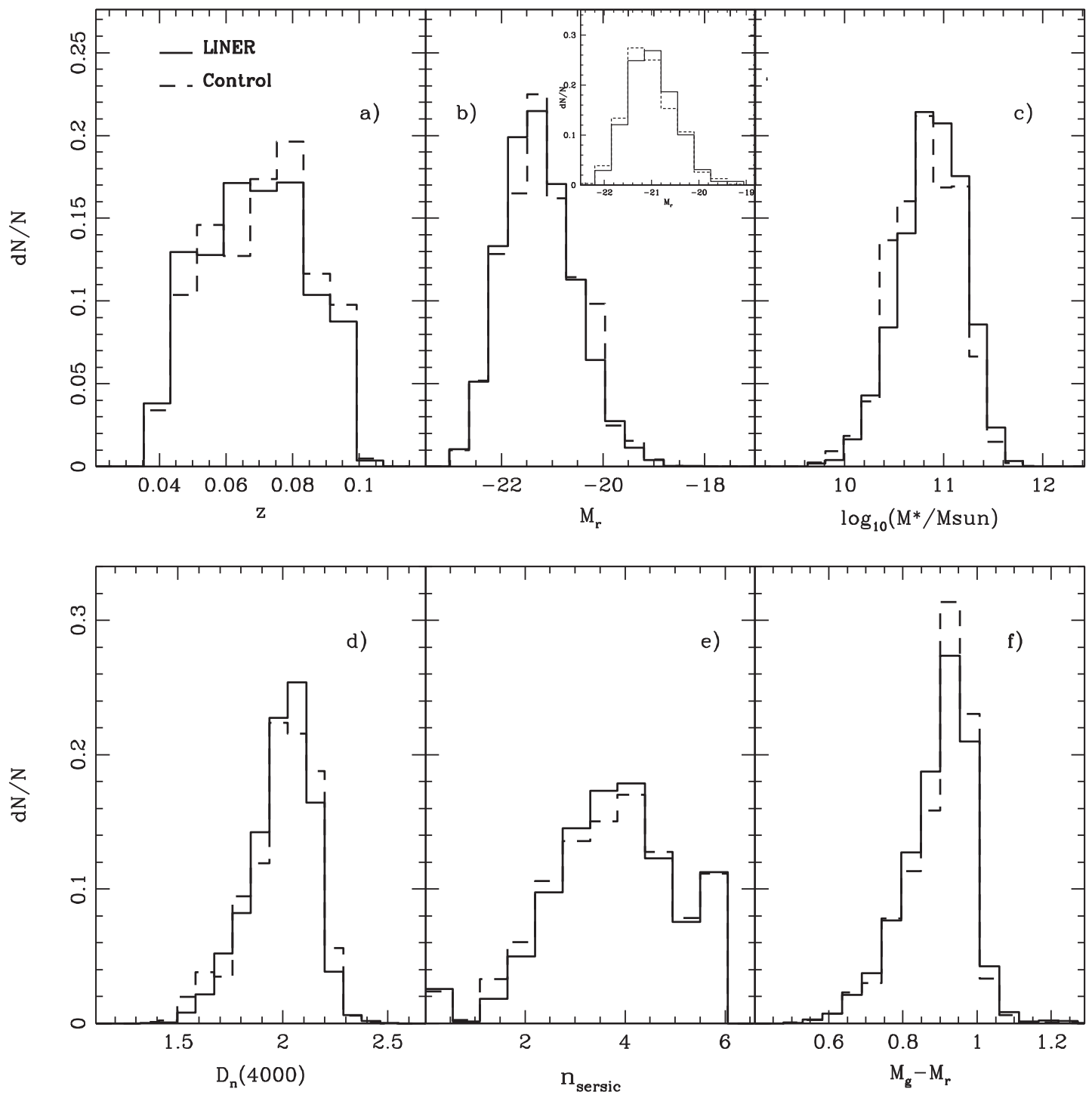

Figure 3. Normalized distributions of galaxy properties for LINERs (solid line) and control samples (dashed) belonging to galaxy groups within $r_{\mathrm{p}}<2 r_{\mathrm{vir}}$ and $\Delta V<1000 \mathrm{~km} \mathrm{~s}^{-1}$. The inner box in panel (b) corresponds to the luminosity distribution of the brightest member of galaxy groups matched in both samples.

within the dark matter halo of the groups and, also, increasing the sample to obtain more confident statistical results. In this way, finally, we obtained a sample of 3967 LINER galaxies (83 per cent of the total sample) hosted by galaxy groups with more than four members.

To study the true environmental dependence of LINER properties, it is very important to select an appropriate control sample of galaxies without low-ionization emission lines that belong to galaxy groups and fulfil the selection criterion mentioned above. This type of control samples has been used in a series of papers (Coldwell \& Lambas 2003, 2006; Coldwell et al. 2009, 2014) to understand the behaviour of AGNs with respect to non-active galaxies. In addition, by using SDSS mock galaxy catalogues built from the Millennium Simulation, Perez, Tissera \& Blaizot (2009) showed that a suitable control sample for galaxies in pairs should be selected (at least) with matched distributions of redshift, morphology, stellar mass and local density environment. This criterion is also applicable to the case of control galaxies for LINERs. Thus, we construct our control sample by selecting galaxies without low-ionization emission features from SDSS with matched distributions of those five parameters.

It is important to mention that the control sample has been taken from a galaxy sample satisfying the criterion to be closer than $r_{\mathrm{p}}<2$ $r_{\text {vir }}$ and $\Delta V<1000 \mathrm{~km} \mathrm{~s}^{-1}$ with respect to the galaxy group centre. Moreover, any galaxy classified as AGN or LINER has not been included in the control sample, which comprises 3841 galaxies. Fig. 3 shows the normalized distribution of properties for LINER host galaxies within the dark matter haloes of galaxy groups, and their respective control samples. We can appreciate that the control sample suitably reproduces the properties of LINER objects. Both samples are redder, older and more massive than the rest of the galaxies in the SDSS catalogue, and have a Sérsic index arithmetic mean of $n=4$.

In addition, we analysed the fraction of both LINER and control samples that correspond to the central galaxy of groups, identified as the brightest member galaxy. We found a similar percentage of central galaxies for control (16.4 per cent) and LINER (13.8 per cent) samples. The inner box in Fig. 3(b) shows that the luminosity 


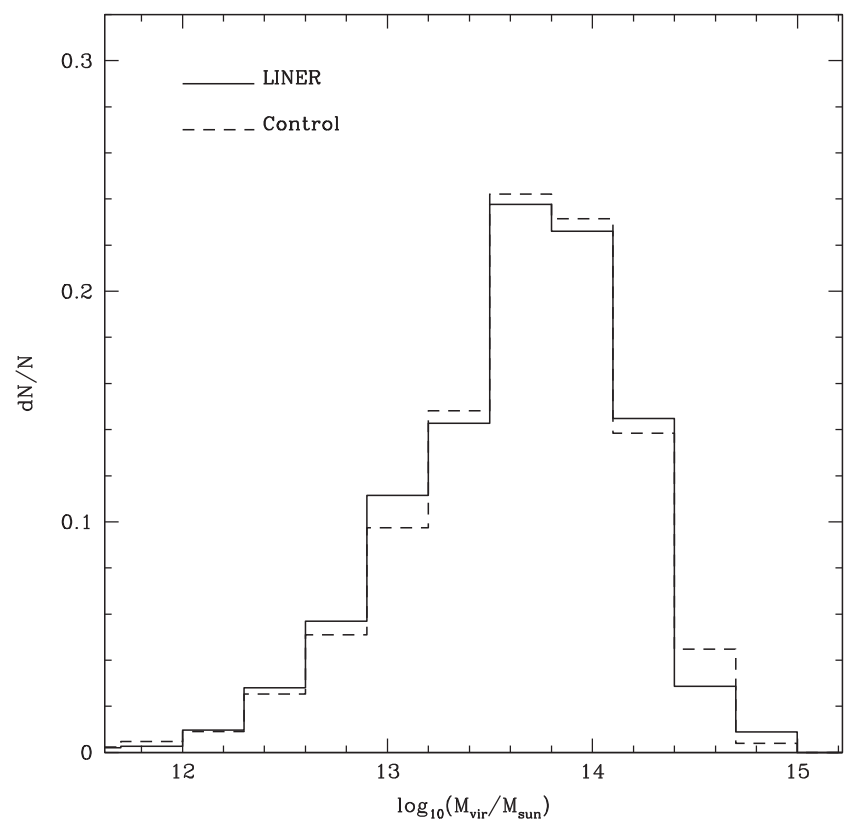

Figure 4. Virial mass distributions for galaxy groups closer than $r_{\mathrm{p}}<2 r_{\mathrm{vir}}$ and $\Delta V<1000 \mathrm{~km} \mathrm{~s}^{-1}$ to LINERs (solid line) and control samples (dashed line).

distribution of the central galaxies in both samples is very similar. Moreover, in Fig. 4, we also compare the virial mass distribution of groups close $\left(r_{\mathrm{p}}<2 r_{\mathrm{vir}}\right.$ and $\left.\Delta V<1000 \mathrm{~km} \mathrm{~s}^{-1}\right)$ to galaxies of our LINER and control samples. The agreement between both distributions shows that, on average, LINER and control galaxies belong to galaxy groups of similar virial mass. As we will see in Section 4, this supports the idea that the difference in their fractions (Figs 6 and 7) could be driven mainly by the presence of low-ionization emission in LINER hosts.

\subsection{Host galaxy properties: dependence on the galaxy group proximity}

The similarity between LINER and control sample properties is what enables us to explore how these are related to the galaxy group parameters with the aim to detect some distinctive features for LINERs. Particularly, the galaxy colours provide an indirect constraint on the evolutionary history of galaxies since other galaxy parameters, for example, morphology and age, affect galaxy colours, resulting in a very appropriate topic for this study.

As in previous works (Coldwell \& Lambas 2006; Coldwell et al. 2009, 2014), we use colours and the age indicator to study the fraction of host galaxies redder than $M_{g}-M_{r}>0.89$ and $D_{\mathrm{n}}(4000)>1.99$, as a function of the galaxy group centre distance and the virial mass. These thresholds for the fractions correspond to mean values of $M_{g}-M_{r}$ and $D_{\mathrm{n}}(4000)$ for the LINER and control samples (see the distributions in Fig. 3). The error bars are calculated with the bootstrap error resampling technique (Barrow, Bhavsar \& Sonoda 1984).

It is possible to observe a slightly higher fraction of red and old galaxies closer to the geometric group centre, as shown in Fig. 5. In addition, the trends of LINER and control samples are nearly indistinguishable. Moreover, other than a weak excess of red galaxies (still within the error bars), we do not find any significant difference with respect to the group virial mass. The conclusion is that the properties of LINER host galaxies at a given distance or

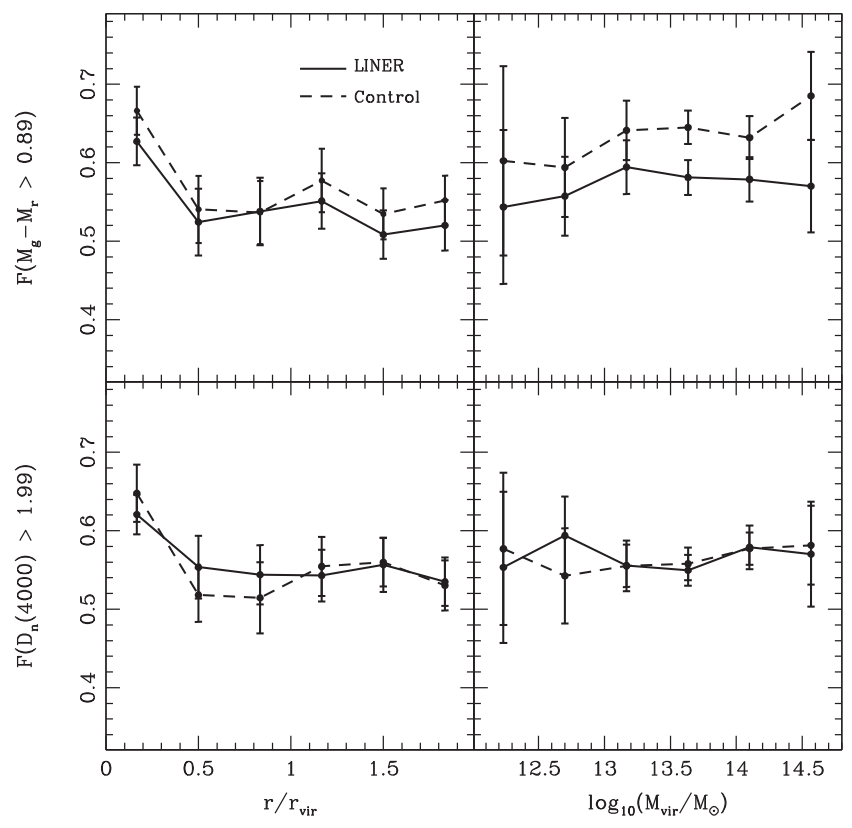

Figure 5. Fraction of red $\left(M_{g}-M_{r}>0.89\right)$ and old $\left[D_{\mathrm{n}}(4000)>1.99\right.$ ] galaxies as a function of the normalized projected distance to the group centre (left-hand panels) and to the galaxy group virial mass (right-hand panels). The solid line corresponds to the LINER sample and the dashed line to the control sample.

virial mass do not vary with respect to control non-active galaxies, so the location within the galaxy group environment does not seem to affect colours and/or ages of LINERs.

\section{OCCURRENCE OF LINERS IN GALAXY GROUPS}

In this section, we explore the probability of finding LINERs with respect to the control sample and the dependence on galaxy group properties such as the virial mass, $M_{\text {vir }}$ (as derived from the virial theorem using the virial radius and velocity dispersion of group members). We also considered the group luminosity, $M_{r 4}$, calculated by adding the $r$-band luminosities of the four brightest galaxy members in each group. This quantity, $M_{r 4}$, is a better proxy for the true underlying group mass than the virial estimate (Eke et al. 2004; Padilla et al. 2004). In this way, we have considered two independent estimates of the group richness for this analysis.

Fig. 6 shows the fraction of both samples with respect to the total number of objects for a given bin of virial mass in logarithmic scale and group luminosity. We observe that the fraction of LINERs drops with the group virial mass and luminosity, while the fraction of control galaxies increases with these parameters. Precisely for the control sample, this is expected due to the morphology-density relation and the sample distributions shown in Fig. 3. Yet somewhat surprisingly, the relation for LINER galaxies is in fact the opposite, even when their host galaxies are matched to the control sample.

This tendency is more significant in the left-hand panel of Fig. 6, where the fraction of galaxies in the brightest groups in the control sample is approximately two times the LINER fraction. At low luminosities, the effect is reversed as the proportion of LINERs reaches its maximum value ( $\sim 56$ per cent) and the difference with respect to the control falls to $\sim 13$ per cent. A comparable trend is observed in the right-hand panel of Fig. 6, with respect to $M_{\text {vir }}$, although the excess of control galaxies with respect to LINERs at 


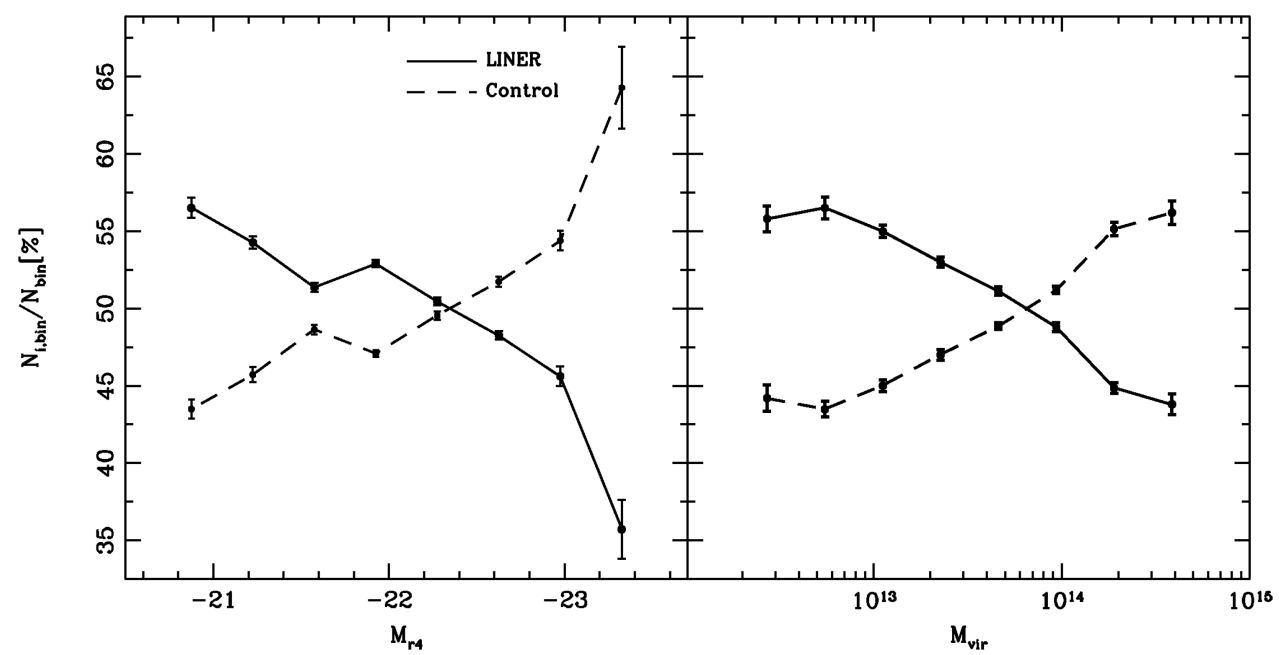

Figure 6. Fraction of LINER (solid line) and the respective control samples (dashed lines) as a function of the group virial mass $M_{\text {vir }}$ (right-hand panel) and brightness $M_{r 4}$ (left-hand panel).

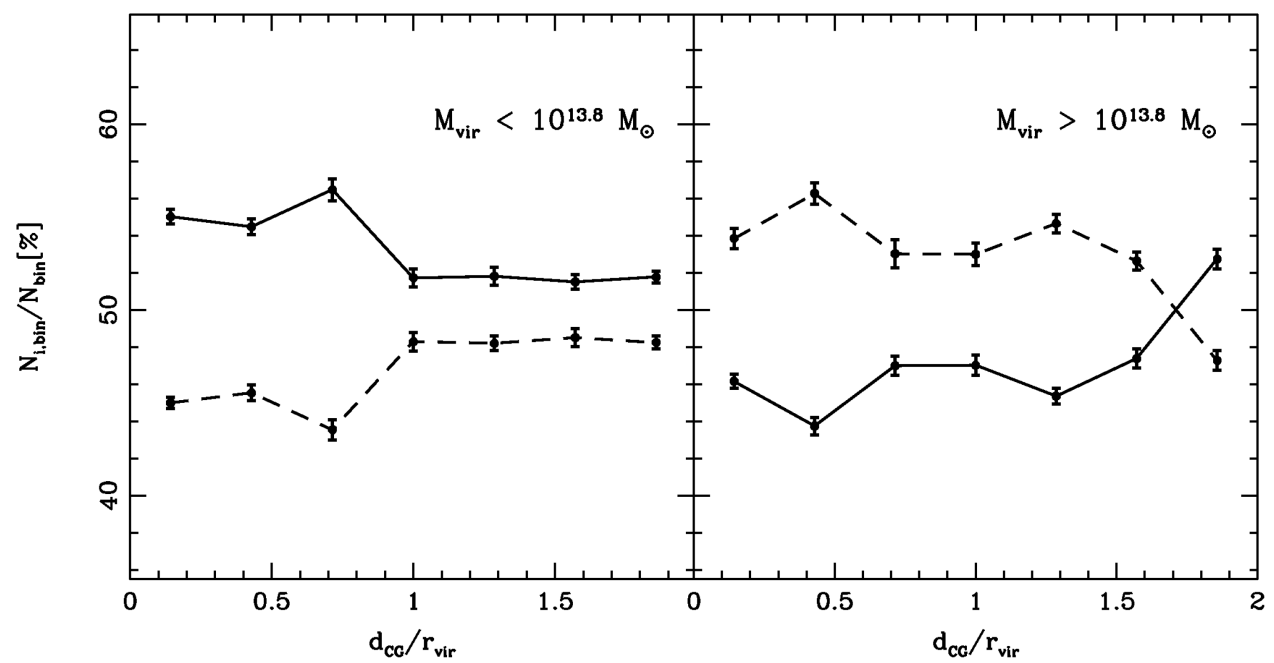

Figure 7. Fraction of LINER (solid line) and the respective control samples (dashed lines) as a function of the normalized distance to the centre of galaxy groups with virial mass $\log _{10}\left(M_{\text {vir }}\right)<13.8$ (left-hand panel) and $\log _{10}\left(M_{\mathrm{vir}}\right) \geq 13.8$ (right-hand panel).

higher values of $M_{\mathrm{vir}}$ is somewhat lower than that shown for the group luminosity, $M_{r 4}$. These results indicate that LINER galaxies have a preference to populate low-mass, low-luminosity environments formed by a higher proportion of blue galaxies with high gas content.

Bearing in mind the fact that several authors have found that the association between active galaxies and environments does not agree with the expected morphology-density relation (Popesso \& Biviano 2006; Coldwell et al. 2009; Padilla 2010; Coldwell et al. 2014), these results suggest that the accretion of material inside the black hole should not be neglected as the main mechanism responsible for the low-ionization emission.

Additionally, it is useful to perform this analysis by discriminating groups with a group mass indicator. Fig. 7 shows the fraction of LINER and control galaxies as a function of the distance to the galaxy group centre, normalized to the virial radius, $d_{\text {cg }} / r_{\text {vir }}$, for rich groups $\left(\log _{10} M_{\text {vir }}>13.8 \mathrm{M}_{\odot}\right)$ and for poor groups $\left(\log _{10} M_{\text {vir }}<13.8 \mathrm{M}_{\odot}\right)$. In poor groups, we observe a higher fraction of LINERs with respect to the control sample, which is not unexpected, as shown in Fig. 6. This effect is almost independent of scale with only a weak increase in the fraction of LINER galaxies towards the inner regions of poor galaxy groups. In rich groups, we find that the fraction of LINERs increases significantly towards the outer limits of these systems and that the fraction of LINER objects is lower than that in control galaxies. Therefore, this reinforces the hypothesis that LINER galaxies prefer to populate low-density environments, where the probability of major mergers rises due to low galaxy velocity dispersions favouring this type of interactions. This seems to be in agreement with previous results for AGNs found in the literature (Popesso \& Biviano 2006; Alonso et al. 2007; Coldwell et al. 2009).

\section{DISCUSSION}

From SDSS-DR7 data, we derived a large LINER catalogue at redshifts $0.04<z<0.1$. As only a small fraction of LINERs are hosted in groups of galaxies, we adopted LINER objects with $r_{\mathrm{p}}<2 r_{\text {vir }}$ and $\Delta V<1000 \mathrm{~km} \mathrm{~s}^{-1}$ as belonging to galaxy groups. In this way, our effective LINER sample comprises 3967 objects, distributed in 2879 groups. The mean number of LINERs per group 
is $\sim 1.4$. In order to uncover the true environmental dependence of the characteristics of galaxies hosting LINERs and get a hint on the process responsible for generating low-ionization emission lines, we constructed a control sample of non-active galaxies matched in redshift, luminosity, age, stellar mass, morphology and colour. We also verified that (1) the percentage of LINER and control galaxies corresponding to the central members of groups is quite similar between both samples; and (2) galaxy groups nearby both samples have similar virial masses.

Following previous work, we have explored how host properties depend on mass and the proximity to galaxy groups. We have calculated the fraction of red $\left(M_{g}-M_{r}>0.89\right)$ and old [ $\left.D_{\mathrm{n}}(4000)>1.99\right]$ galaxies with respect to the normalized projected distance, $d_{\mathrm{cg}} / r_{\mathrm{vir}}$, to the geometric group centre, and to the virial mass, $M_{\mathrm{vir}}$. We find no significant difference in the tendencies of LINER and control samples. Therefore, the properties of hosts in both samples do not seem to vary with respect to the location or mass of the galaxy groups.

In addition, we have carried out a detailed analysis the LINER occurrence within galaxy group haloes and quantified the colour and age dependence with respect to representative galaxy group parameters such as radius, virial mass and group luminosity. We study the fraction of LINERs with respect to two independent parameters representative of the galaxy group mass $\left(M_{\mathrm{vir}}\right.$ and $\left.M_{r 4}\right)$. The results show a strong difference between LINER and control galaxies. While the latter fraction increases for rich groups, the fraction of LINERs drops drastically. This effect is more significant for the highest luminosity groups, where we find about two times more control than LINER galaxies, indicating an evident preference of LINERs to inhabit low-mass galaxy groups. Besides, LINER galaxies do not seem to follow the expected morphology-density relation in the proximity of rich galaxy groups. Such a behaviour of LINERs agrees with that corresponding to active objects (Seyfert 2, quasars, etc.) analysed in previous works. So, although we have no certainty about the degree at which the mechanisms are responsible of the low-ionization emission, the presence of nuclear activity is certainly involved and should not be discarded.

In conclusion, we have shown the higher probability of LINERs to be found in lower density environments, such as poor groups, where the galaxy interactions and content of gas are more abundant. Thus, the global conditions of these environments could favour the presence of the LINER emission.

\section{ACKNOWLEDGEMENTS}

We would like to thank the anonymous referee for the comments that helped to improve this paper. This work was supported in part by the Consejo Nacional de Investigaciones Científicas y Técnicas de la República Argentina (CONICET), in part by the Consejo Nacional de Investigaciones Científicas, Técnicas y de Creación Artística de la Universidad Nacional de San Juan (CICITCA) and in part by the Secretaría de Estado de Ciencia, Tecnología e Innovación del Gobierno de San Juan (SECITI).

Funding for the SDSS and SDSS-II has been provided by the Alfred P. Sloan Foundation, the Participating Institutions, the National Science Foundation, the US Department of Energy, the National Aeronautics and Space Administration, the Japanese Monbukagakusho, the Max Planck Society and the Higher Education Funding Council for England. The SDSS website is http://www.sdss.org/. The SDSS is managed by the Astrophysical Research Consortium for the Participating Institutions. The Participating Institutions are the American Museum of Natural History, the Astrophysical
Institute Potsdam, the University of Basel, the University of Cambridge, the Case Western Reserve University, the University of Chicago, Drexel University, Fermilab, the Institute for Advanced Study, the Japan Participation Group, Johns Hopkins University, the Joint Institute for Nuclear Astrophysics, the Kavli Institute for Particle Astrophysics and Cosmology, the Korean Scientist Group, the Chinese Academy of Sciences (LAMOST), the Los Alamos National Laboratory, the Max Planck Institute for Astronomy (MPIA), the Max Planck Institute for Astrophysics (MPA), the New Mexico State University, the Ohio State University, the University of Pittsburgh, the University of Portsmouth, the Princeton University, the United States Naval Observatory and the University of Washington.

\section{REFERENCES}

Abazajian K. N. et al., 2009, ApJS, 182, 543

Alonso M. S., Lambas D. G., Tissera P., Coldwell G., 2007, MNRAS, 375, 1017

Baldwin J. A., Phillips M. M., Terlevich R., 1981, PASP, 93, 5 (BPT)

Barrow J. D., Bhavsar S. P., Sonoda B. H., 1984, MNRAS, 210, 19

Bernardi M. et al., 2003, AJ, 125, 1882

Binette L., Magris C. G., Stasinska G., Bruzual A. G., 1994, A\&A, 292, 13

Blanton M. R., Roweis S., 2007, AJ, 133, 734

Blanton M. R. et al., 2003a, ApJ, 592, 819

Blanton M. R. et al., 2003b, ApJ, 594, 186

Blanton M. R., Eisenstein D., Hogg D. W., Schlegel D. J., Brinkmann J., 2005, ApJ, 629, 143

Brinchmann J., Charlot S., White S. D. M., Tremonti C., Kauffmann G., Heckman T., Brinkmann J., 2004, MNRAS, 351, 1151

Calzetti D., Armus L., Bohlin R. C., Kinney A. L., Koornneef J., StorchiBergmann T., 2000, ApJ, 533, 682

Capetti A., Baldi R. D., 2011, A\&A, 529, 126

Coldwell G. V., Lambas D. G., 2003, MNRAS, 344, 156

Coldwell G. V., Lambas D. G., 2006, MNRAS, 371, 786

Coldwell G. V., Lambas D. G., Söchting I. K., Gurovich S., 2009, MNRAS, 399, 88

Coldwell G. V., Gurovich S., Diaz Tello J., Söchting I. K., Lambas D. G., 2014, MNRAS, 437, 1199

Domínguez M., Muriel H., Lambas D. G., 2001, AJ, 121, 1266

Dopita M. A., Sutherland R. S., 1995, ApJ, 455, 468

Dressler A., 1980, ApJ, 236, 351

Dressler A., 1984, ARA\&A, 22, 185

Dressler A. et al., 1997, ApJ, 490, 577

Dudik R. P., Satyapal S., Marcu D., 2009, ApJ, 691, 1501

Eke V. R. (The 2dFGRS Team) et al., 2004, MNRAS, 355, 769

Filippenko A. V., Terlevich R., 1992, ApJ, 397, L79

González-Martín O., Masegosa J., Márquez I., Guainazzi M., JiménezBailón E., 2009a, A\&A, 506, 1407

González-Martín O., Masegosa J., Márquez I., Guainazzi M., 2009b, ApJ, 704, 1570

Goudfrooij P., Hansen L., Jorgensen H., Norgaard-Nielsen H., 1994, A\&AS, 105,341

Graves G. J., Faber S. M., Schiavon R. P., Yan R., 2007, ApJS, 671, 243

Groves B. A., Dopita M. A., Sutherland R. S., 2004, ApJS, 153, 75

Heckman T. M., 1980, A\&A, 87, 152

Ho L. C., Filippenko A. V., Sargent W. L. W., 1997, ApJS, 112, 391

Ho L. C., Filippenko A. V., Sargent W. L. W., 2003, ApJ, 583, 159

Hogg D. W. et al., 2004, ApJ, 601, 29

Huchra J. P., Geller M. J., 1982, ApJ, 257, 423

Kauffmann G., Haehnelt M. G., 2002, MNRAS, 332, 529

Kauffmann G. et al., 2003, MNRAS, 341, 33

Kewley L. J., Dopita M. A., Sutherland R. S., Heisler C. A., Trevena J., 2001, ApJ, 556, 121

Kewley L. J., Groves B., Kauffmann G., Heckman T. M., 2006, MNRAS, 372,961 
Masegosa J., Márquez I., Ramirez A., González-Martín O., 2011, A\&A, 527,23

Merchán M., Zandivarez A., 2005, ApJ, 630, 759

Nagar N. M., Falcke H., Wilson A. S., 2005, A\&A, 435, 521

Osterbrock D. E., Miller J. S., eds, 1989, Proc. IAU Symp. 134, Active Galactic Nuclei. Kluwer, Dordrecht

Padilla N. et al., 2004, MNRAS, 352, 211

Padilla N., Lambas D. G., González R., 2010, MNRAS, 409, 936

Perez J., Tissera P., Blaizot J., 2009, MNRAS, 397, 748

Petrosian V., 1976, ApJ, 209, 1

Phillip M. M., Jenkins C. R., Dopita M. A., Sadler E. M., Binette L., 1986, AJ, 91, 1062

Popesso P., Biviano A., 2006, A\&A, 460, L23

Sarzi M. et al., 2010, MNRAS, 402, 2187
Sérsic J. L., 1963, Bol. Asociacion Argentina Astron., 6, 41

Sodré L., Stasińska G., 1999, A\&A, 345, 391

Stasínska G., Vale Asari N., Cid Fernandes R., Gomes J. M., Schlickmann M., Mateus A., Schoenell W., Sodré L., Jr2008, MNRAS, 391, 29

Strauss M. et al., 2002, AJ, 124, 1810

Toomre A., Toomre J., 1972, ApJ, 178, 623

Tremonti C. et al., 2004, ApJ, 613, 898

Yan R., Blanton M.R, 2012, ApJ, 747, 61

Yan R., Newman J. A., Faber S. M., Konidaris N., Koo D., Davis M., 2006, ApJ, 648, 821

Zapata T., Pérez J., Padilla N., Tissera P., 2009, MNRAS, 394, 2229

This paper has been typeset from a $\mathrm{T}_{\mathrm{E}} \mathrm{X} / \mathrm{L} \mathrm{T} \mathrm{E} \mathrm{X}$ file prepared by the author. 